\title{
Frequent nocturnal awakening in early life is associated with nonatopic asthma in
} children

\author{
A.L. Kozyrskyj*,\#, G.E. Kendall ${ }^{\uparrow,+, \S}$, S.R. Zubrick ${ }^{\uparrow, \S, f}$, J.P. Newnham** and P.D. Sly ${ }^{\dagger, \S}$
}

ABSTRACT: Sleep deprivation has become a common phenomenon of the Western world and is associated with a variety of medical problems in children. This retrospective longitudinal analysis of a community-based birth cohort was undertaken to determine whether frequent nocturnal awakening during early life was associated with the development of childhood asthma.

2,398 children born to mothers recruited from the antenatal clinics of a single hospital in Perth, Australia during 1989-1991 were followed up at years 1, 2, 3, 6, 8, 10 and 14. Parent-completed questionnaires were analysed. The odds ratio for asthma at age 6 and 14 yrs in children with frequent nocturnal awakening during the first 3 yrs after birth was determined from multiple logistic regression.

Following adjustment for asthma risk factors, co-sleeping and family stress, persistent nocturnal awakening was associated with nonatopic asthma at age 6 and 14 yrs (at age 14 yrs: OR $2.18,95 \% \mathrm{Cl} 1.15-4.13$ ) but not with atopic asthma.

We found an increased risk of nonatopic asthma in children following frequent nocturnal awakening during the first 3 yrs of life. These hypothesis-generating data suggest the need for further systematic study of the effects of disordered sleep in early life on the development of asthma.

KEYWORDS: Asthma, child, infant, Raine study, sleep

leep deprivation is a common phenomenon of the Western world; normal sleep duration has decreased from $9 \mathrm{~h}$ in 1910 to an average of 7.5 hours in the 1970s [1]. Inadequate sleep is an issue in young children. One third of American children experience one night or more of insufficient sleep weekly [2]. Disordered sleep has been linked to a variety of medical problems in children, including hyperactivity disorders $[3,4]$. Short duration of sleep has also been associated with decreased cortisol reactivity to stress [5] and being overweight in adolescence [6, 7].

Sleep is important for growth during childhood and maturation of the hypothalamic-pituitaryadrenal (HPA) axis [8, 9]. Higher morning cortisol and lower growth hormone levels have been observed in sleep-deprived infants and children [10-12]. Shorter stature is more common in children with asthma [13]. Infants at risk for allergic disease have exaggerated cortisol responses to stress, while asthmatic schoolchildren have lower HPA reactivity [14-16]. The co-existence of these phenomena in childhood asthma raises the possibility that sleep deprivation may play a role in asthma development. More recently, higher rates of asthma in the inner city have been attributed to the detrimental effects of noise on sleep in children [17].

Cross-sectional surveys have found sleep problems to be more prevalent in schoolchildren with atopic disease, with the strongest association reported for hay fever or atopic dermatitis $[2,18]$. While causal links between infant sleep and childhood asthma are plausible, this hypothesis has not been tested. Our primary research objective was to determine whether frequent nocturnal awakening during early life was associated with the development of asthma in a community-based, longitudinal birth cohort of children. A secondary research objective was to test the association with the asthma phenotypes: atopic and nonatopic asthma.

\section{MATERIALS AND METHODS}

The study population came from the Western Australian Pregnancy Cohort (Raine) Study of children born in 1989-1991 of mothers enrolled at

\section{AFFILIATIONS}

*Dept of Paediatrics, University of Alberta, Edmonton, $A B$,

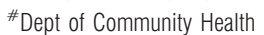
Sciences, Dept of Paediatrics and Child Health, University of Manitoba, Winnipeg, MB, Canada.

TTelethon Institute for Child Health Research,

${ }^{+}$School of Nursing and Midwifery, Curtin University of Technology,

${ }^{f}$ Centre for Developmental Health, Curtin University of Technology,

${ }^{\S}$ Centre for Child Health Research,

University of Western Australia, and

**School of Women's and Infants'

Health, University of Western

Australia, Perth, Australia.

\section{CORRESPONDENCE}

A.L. Kozyrskyj

8226a Aberhart Centre

11402 University Ave

Edmonton

AB T6G 2J3

Canada

E-mail: kozyrsky@ualberta.ca

Received:

March 122009

Accepted after revision:

Aug 122009 
gestational age 16-20 weeks from antenatal clinics at the main tertiary maternal hospital or nearby practices in Perth, Australia [19]. Mothers were not recruited on the basis of a history of asthma or allergy, and were broadly representative of the females attending the hospital. Children were followed up at years $1,2,3,6,8,10$ and 14 . The likelihood of asthma at age 6 and 14 yrs subsequent to parental report of sleep during the first 3 yrs of life was determined. This study was approved by the institutional ethics committee.

Study measures were obtained from parent-completed questionnaires. Complete information on infant sleep at $1 \mathrm{yr}$ was available for 2,398 children. There was a loss to follow-up or missing data on asthma status for $399(17 \%)$ children at age 6 and for $705(29 \%)$ children at age 14 yrs. Asthma risk or protective factors, such as maternal history of asthma, maternal smoking, breastfeeding for $<6$ months and dog ownership, were more prevalent in excluded children. Mothers of children with missing data were also more likely to be single parents, to have a lower level of education and to experience high stress.

Each year for the first 3 yrs after birth, parents answered questions about the frequency of night awakenings in their child in the past week. Frequent nocturnal awakening was defined as parental report of their child waking during the night more than twice weekly. Children who awoke twice a week or less were defined as having normal sleep. Parent assessment of night awakenings is well correlated with sleep duration in children and other objective measures of sleep [20-22]. SADEH [23] found significant correlations between parent-reported night awakenings in infants over the past week, sleep duration and actigraph measurements of movement during sleep. A measure of persistent nocturnal awakening was created on the basis of parent report of frequent nocturnal awakening each year over the 3-yr period or during the first and third years after birth. This 3-yr time period coincides with the development of adult patterns of sleeping and circadian regulation of the HPA axis $[9,12]$. Children with frequent nocturnal awakening at age $1 \mathrm{yr}$, but with missing values for nocturnal awakening at age 3 yrs, were conservatively assigned to the category of no persistent nocturnal awakening $(\mathrm{n}=134)$.

Current asthma at age 6 and age 14 yrs was defined using a strict definition that required the presence of all three of the following: ever being diagnosed with asthma by a physician; asthma symptoms (wheeze or nocturnal cough) in the past 12 months; and current use of asthma medications (controller or reliever drugs) [24-26]. Children in the Raine cohort meeting this definition of current asthma are reported to have significant deficits in lung function and greater sensitivity to the methacholine challenge test for bronchial hyperreactivity [24]. Atopic asthma was defined as a positive skin test (wheal size $\geqslant 3 \mathrm{~mm}$ to common aeroallergens) in children with current asthma.

Risk and protective factors for asthma included sex, household income, single-parent status, total number of siblings at birth, maternal history of asthma (recorded during pregnancy), maternal smoking during pregnancy, maternal smoking during the first year of life, pre-term birth ( $<37$ weeks), low birth weight status $(<2,500 \mathrm{~g})$, delivery by elective caesarian section, maternal age $<20$ yrs, maternal education less than high school, family stress during pregnancy and the postnatal period (using the validated life events instrument reported by TENNANT and ANDREWS [27]), duration of exclusive breastfeeding and pet ownership during the first year of life.

Crude odds ratios for current asthma at age 6 and 14 yrs, and odds ratios adjusted for the above confounding factors were determined from multiple logistic regression analysis, using SAS software (SAS Institute, Cary, NC, USA). As infant and parent co-sleeping may heighten parent awareness of sleep interruptions, a measure of co-sleeping (sharing a bed with parents) at age $1 \mathrm{yr}$ was added to models. Separate models were tested for male and female children. To test for reverse causation (sleep disruption due to wheezing at night, a marker for potential future asthma), analyses were adjusted for presence of wheeze during the child's sleep in each of the first 3 yrs [28]. Model results were similar for each of the years, but we only report those with wheeze in the first year because this identified the most children. We also re-ran models after excluding 439 children with any wheeze during the first year of life. Variables were retained in models at the $95 \%$ level of confidence, but final results were interpreted against a Bonferroni-corrected p-value of 0.025 to account for multiple testing.

\section{RESULTS}

Among the 2,398 newborns in the Raine cohort study followed at $1 \mathrm{yr}, 39.2 \%$ of infants (mean (range) age: 14 (11-16) months) had frequent nocturnal awakening during the first year of life. $30 \%$ of children awoke occasionally and $31 \%$ of children never awoke during their sleep. The prevalence of frequent nocturnal awakening decreased to $27.2 \%$ by year three (table 1 ). $13.6 \%$ of all children continued to have frequent awakenings at night during the second and third year of life. $5 \%$ of children had new onset frequent nocturnal awakening at age 3 yrs. Children with persistent nocturnal awakening were more likely to sleep with parents or other family members (table 1). As estimated by parents during follow-up visits, infants with night awakenings slept less in later childhood than their peers.

Frequent nocturnal awakening during infancy was more common if mothers experienced high stress during pregnancy or if infants had a greater number of siblings (table 2). It was less common in infants born before 37 weeks' gestation. Persistent nocturnal awakening was more prevalent in households with low income, money problems and high family stress. Both first-year and persistent nocturnal awakening were less prevalent if mothers were $<20$ yrs old or if they smoked during the first year of their child's life, and more prevalent among infants exclusively breastfed for $>6$ months.

$18 \%$ of children had current asthma at age 6 yrs (mean (range) age: 5.9 (5.3-7.0) yrs). Current asthma at age 14 yrs (mean (range) age: 14 (13-15) yrs) was present in $9.4 \%$ of children. One third of children with asthma at age 6 yrs continued to have asthma at age $14 \mathrm{yrs} .55 \%$ of children with asthma at age 6 yrs had a positive skin test, as did almost $60 \%$ of children at age 14 yrs. Asthma at age 6 yrs was more prevalent if children were male, born prematurely or low birth weight; if mothers had asthma, a lower education and were $<20$ yrs old; and if mothers reported stressful events during pregnancy and the first few years of the child's life (table 2).

The prevalence of current asthma at age 6 yrs was similar in children with and without frequent nocturnal awakening at 


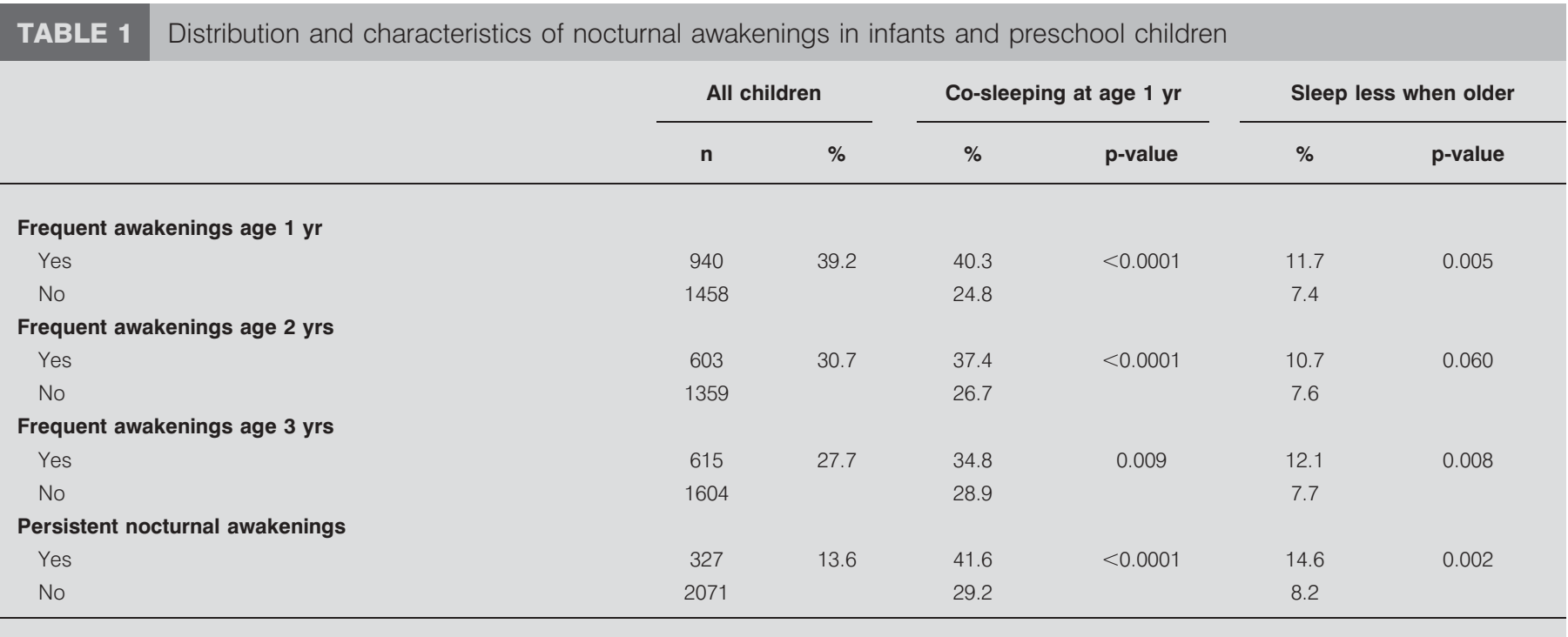

age $1 \mathrm{yr}(18 \%$, unadjusted odds ratio in table 3$)$. Maternal asthma, high family stress, male sex and prematurity were positively associated with asthma at age 6 yrs, and dog ownership lessened the risk of asthma. Adjustment for these factors and co-sleeping did not alter the null association between frequent nocturnal awakening and asthma (table 3). However, asthma prevalence at age 14 yrs was higher (11.3\%) in children with nocturnal awakening than in children without frequent awakenings during the first year of life $(8.4 \% ; \mathrm{p}<0.04$, unadjusted odds ratio in table 4). Maternal asthma, cat ownership and wheeze during sleep were positively associated with asthma at age $14 \mathrm{yrs}$, while single-parent status was protective. Adjustment for these risk factors and co-sleeping (table 4) yielded a significant association between frequent nocturnal awakening and asthma (adjusted OR 1.42, 95\% CI 1.02-1.98). However, the association became nonsignificant when adjusted for high family stress.

Persistent nocturnal awakening was not associated with increased risk of asthma at age 6 yrs in unadjusted associations or following adjustment for co-sleeping, wheeze during sleep, family stress, maternal asthma, prematurity, male sex and dog ownership (table 3). The crude odds ratio between persistent nocturnal awakening and asthma at age 14 was 1.66 (95\% CI 1.11-2.48; table 4). Following adjustment for maternal asthma, cat ownership and co-sleeping, the strength of the association was unchanged (adjusted OR 1.77, 95\% CI 1.12-2.67) and remained statistically significant following further adjustment for wheeze during sleep (adjusted OR 1.81) and for family stress (adjusted OR 1.72).

The association with persistent nocturnal awakening was stronger for nonatopic asthma at age 14 yrs, ranging from an unadjusted OR of 2.04 to a fully adjusted OR of 2.18 (95\% CI 1.15-4.13; table 4). This association was also seen with nonatopic asthma at age 6 yrs (OR 1.87, 95\% CI 1.08-3.25; table 3). No associations were found with atopic asthma at either age (for atopic asthma at age 14 yrs: OR 1.27, 95\% CI 0.73-2.20). Sensitivity analyses in which 134 children with frequent nocturnal awakening at age $1 \mathrm{yr}$, but missing values at age 3 yrs, were excluded, yielded similar associations with asthma or nonatopic asthma at age 14 yrs. Finally, following the exclusion of 439 children with any wheeze during the first year of life, the association between persistent nocturnal awakening and asthma at age 6 or 14 yrs also remained (tables 3 and 4). The p-value for the association with nonatopic asthma at age 6 yrs was improved in the sensitivity analyses. All of the sensitivity analyses for nonatopic asthma at age 14 yrs were at a p-value below the Bonforenni corrected value of $p=0.025$. There was no association between new onset frequent nocturnal awakening sleep at age 3 yrs and asthma development.

\section{DISCUSSION}

The results of this retrospective analysis of a community-based cohort of children born in Western Australia in the early 1990s document an association between parental report of frequent nocturnal awakening in early life and nonatopic asthma in childhood. If frequent nocturnal awakening was present in the first year of life and continued for the following 2 yrs, the risk of nonatopic asthma was doubled (OR 2.18, 95\% CI 1.15-4.13) at age 14 yrs. This association was independent of co-sleeping, wheezing during the night and many well-known early life risk factors for asthma. No association was seen between frequent nocturnal awakening and atopic asthma. While shorter duration of sleep has been linked to hyperactivity disorders and obesity in children, to the best of our knowledge, this is the first report of frequent nocturnal awakening in early life and asthma development $[4,6,7]$.

Our findings have biological plausibility. Several pro-inflammatory cytokines, such as interleukin (IL)-6, IL-1 and tumour necrosis factor (TNF)- $\alpha$, participate in sleep control [29-31]. Two of these cytokines, IL- 6 and TNF- $\alpha$, are elevated in sleepdeprived adults [32]. Sleep disturbances during pregnancy have also been associated with increases in Il-6 and TNF- $\alpha$ [33, 34]. TNF- $\alpha$ has been implicated in neutrophilic inflammation in adults with severe asthma [35]; neutrophilic airway inflammation has been observed in children with sleep apnoea [36]. Higher TNF- $\alpha$ serum levels are seen following viral wheeze in infants and bronchial hyperactivity in nonatopic children [35, $37,38]$. The neuropeptides adenosine and hyposecretins are also 
TABLE 2 Distribution of frequent nocturnal awakenings and asthma by potential confounding factors

\begin{tabular}{|c|c|c|c|c|c|c|}
\hline & \multicolumn{2}{|c|}{ Frequent awakenings age $1 \mathrm{yr}$} & \multicolumn{2}{|c|}{$\begin{array}{l}\text { Persistent nocturnal } \\
\text { awakenings }\end{array}$} & \multicolumn{2}{|c|}{ Asthma at age 6 yrs } \\
\hline & $\%$ & p-value & $\%$ & p-value & $\%$ & p-value \\
\hline \multicolumn{7}{|c|}{ Mother was single-parent when age 1 or $3 \mathrm{yrs}^{\#}$} \\
\hline Yes & 36.1 & & 19.1 & 0.06 & 21.0 & \\
\hline Yes & 39.8 & & 16.6 & 0.01 & 20.6 & 0.030 \\
\hline No & 37.9 & & 12.5 & & 16.7 & \\
\hline \multicolumn{7}{|c|}{ Household income $<25,000$ AUD when age 1 or $3 \mathrm{yrs}^{\#}$} \\
\hline Yes & 39.5 & & 18.4 & 0.02 & 17.3 & \\
\hline No & 39.2 & & 13.1 & & 18.1 & \\
\hline Yes & 45.7 & 0.02 & 17.5 & 0.05 & 25.8 & 0.001 \\
\hline No & 38.4 & & 13.2 & & 17.1 & \\
\hline \multicolumn{7}{|c|}{ Stressful events when age 1 or $3 \mathrm{yrs}^{\#}$} \\
\hline Yes & 40.5 & & 20.4 & 0.01 & 24.7 & 0.0001 \\
\hline No & 38.2 & & 14.9 & & 16.0 & \\
\hline \multicolumn{7}{|c|}{ Mother smoked during her pregnancy } \\
\hline Yes & 34.2 & $<0.0001$ & 12.2 & 0.07 & 20.0 & 0.08 \\
\hline No & 43.1 & & 14.9 & & 16.9 & \\
\hline \multicolumn{7}{|c|}{ Mother smoked when age $1 \mathrm{yr}$} \\
\hline Yes & 32.9 & $<0.0001$ & 11.1 & 0.02 & 20.2 & 0.08 \\
\hline No & 41.9 & & 14.8 & & 16.9 & \\
\hline \multicolumn{7}{|c|}{ Maternal age $<\mathbf{2 0}$ yrs at birth } \\
\hline No & 39.3 & & 14.1 & & 16.0 & \\
\hline \multicolumn{7}{|c|}{ Number of siblings at birth } \\
\hline $2+$ & 47.9 & 0.03 & 16.6 & & 13.5 & \\
\hline 1 & 41.5 & & 14.1 & & 20.6 & \\
\hline 0 & 38.1 & & 13.3 & & 18.0 & \\
\hline \multicolumn{7}{|c|}{ Premature birth ( $<37$ weeks) } \\
\hline Yes & 33.1 & 0.03 & 10.2 & 0.09 & 25.9 & 0.001 \\
\hline No & 40.0 & & 14.1 & & 17.2 & \\
\hline \multicolumn{7}{|c|}{ Low birth weight $(<2500 \mathrm{~g})$} \\
\hline Yes & 30.0 & & 3.3 & & 33.3 & 0.04 \\
\hline No & 39.4 & & 13.8 & & 17.9 & \\
\hline \multicolumn{7}{|c|}{ Exclusive breastfeeding $>6$ months } \\
\hline Yes & 44.7 & $<0.0001$ & 16.4 & 0.003 & 16.0 & 0.08 \\
\hline No & 36.1 & & 12.1 & & 19.1 & \\
\hline
\end{tabular}

Only p-values $<0.10$ are reported. ${ }^{\#}$ : for these variables, data on persistent nocturnal awakenings is given for children aged 3 yrs.

elevated during sleep deprivation [29-31]. Vasoactive intestinal peptide (VIP) decelerates rapid eye movement (REM) and nonREM cycles [39]. The neuropeptide VIP plays a role in T-cell activation [31, 40]. These connections between the nervous and immune systems suggest a pathway for the effect of sleep on immune function. However, the present study was not designed 


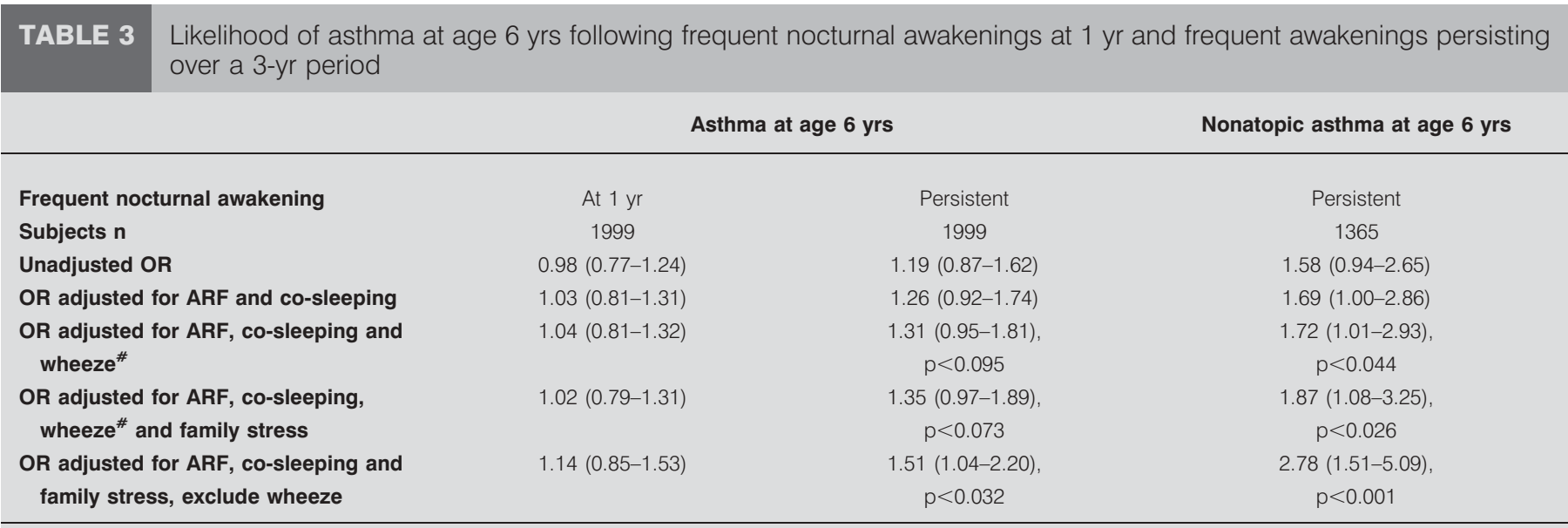

ARF: asthma risk factors. ${ }^{\#}$ : wheeze during sleep.

to investigate the link between nocturnal awakening in infancy and nonatopic asthma, and cannot shed light on the underlying biological mechanisms.

Sleep disruption not only affects the duration of sleep, but also the stages of REM and non-REM sleep [29]. Parent estimates of their child's sleeping hours are highly correlated with sleep time measured in the laboratory [22]. Objective assessments of sleep duration, movement during sleep and night awakenings are also correlated in infants [21, 23]. TOUCHETTE et al. [20] reported that young children with frequent nocturnal awakening slept $1.5 \mathrm{~h}$ less than children without frequent awakenings. In the same study, $10 \%$ of infants with frequent nocturnal awakening continued to have frequent awakenings 2 yrs later. Frequent nocturnal awakening was more likely in the presence of co-sleeping and breastfeeding at night. It has also been found that pre-term infants are less likely to wake at night and that maternal smoking decreases arousability of sleeping infants [41, 42]. The data we present here are remarkably similar to these reports. All of our analyses were adjusted for the presence of co-sleeping with parent. Finally, our analyses identify the first 3 yrs of life as the critical time period for the effects of nocturnal awakening; no associations were observed between later onset frequent nocturnal awakening and asthma. These findings are noteworthy because this is the period during which infants develop adult-like sleep patterns and diurnal variation in cortisol secretion $[9,12]$.

The major strength of our study is that it was not limited to a birth cohort at high risk for asthma, but studied the effect of sleep in a community-based birth cohort exposed to a broad range of pregnancy and early life exposures. We used a definition of asthma which has been previously validated [43]. Further, access to 14 yrs of longitudinal data enabled the temporal study of the association between frequency of nocturnal awakening in early life and two asthma phenotypes, early school-age asthma and asthma in adolescence. Asthma persists to adolescence in only one third of children with early school-age wheeze; persistent asthma is more likely to be atopic [44]. As such, the association between fragmented sleep and asthma which we found in nonatopic children was unexpected. However, this finding gives credence to mechanisms involving sleep deprivation-induced elevations in TNF- $\alpha$. Akin to our findings, HEATON et al. [37] found TNF- $\alpha$

Likelihood of asthma at age $14 \mathrm{yrs}$ following frequent nocturnal awakenings at $1 \mathrm{yr}$ and frequent awakenings persisting over a 3-yr period

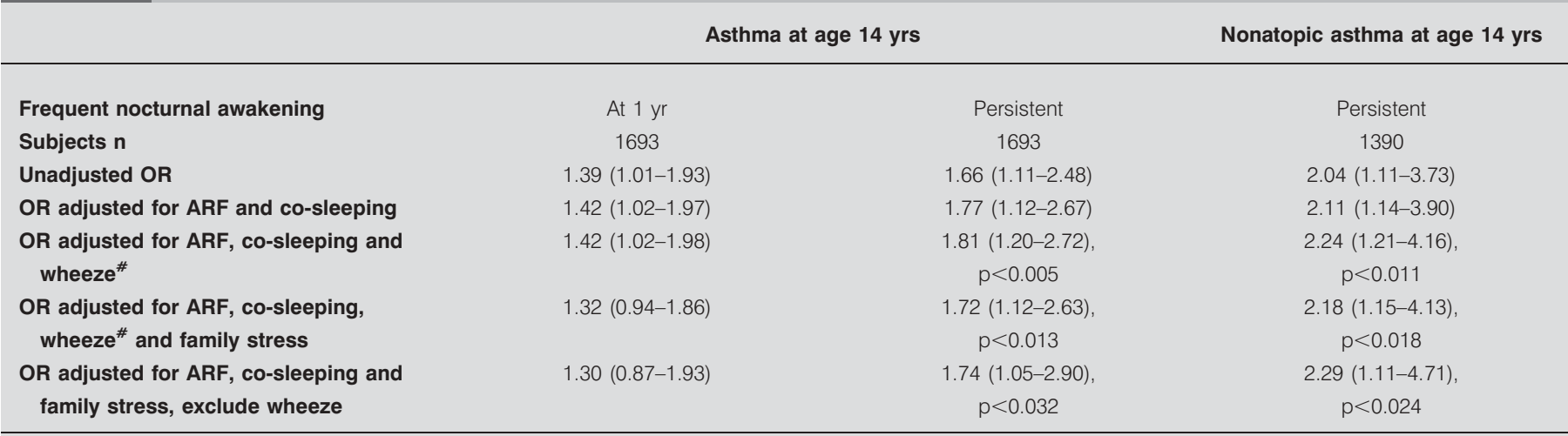

Odds ratios are presented as OR $(95 \% \mathrm{Cl})$. ARF: asthma risk factors. ${ }^{*}$ : wheeze during sleep. 
production from T-cells stimulated with both mitogen and allergens in vitro was elevated in nonatopic children with bronchial hyperreactivity compared with in those without bronchial hyperactivity; no such differences were found among atopic children. Other studies have reported that children with nonatopic asthma are more likely to have recurrent respiratory infections in early life and to have a mother with bronchial hyperactivity $[45,46]$. Further studies will be required in order to determine whether an intrinsic increase in TNF- $\alpha$ responses, potentially as a result of a genetic predisposition, plays a role in the association between poor sleep quality in early life and subsequent nonatopic asthma.

We adjusted our analyses for important confounding factors, such as maternal history of asthma, breastfeeding and pet exposure in early life. Frequent nocturnal awakening and childhood asthma were more common in families with high stress levels. Indeed, family stress explained some of the increased risk of childhood asthma. Asthma and hay fever symptoms, and atopic dermatitis-related pruritis, may be the cause of disrupted sleep in cross-sectional studies of older children $[2,18,47,48]$. We also recognise the possibility that reverse causation may be responsible for our findings in infants because wheeze during infancy can cause nocturnal awakening and is a marker for future asthma [28, 49]. For this reason, we performed two sensitivity analyses: model adjustment for wheeze during the night and exclusion of children with any wheeze during the first year of life. Our findings were unchanged in the sensitivity analyses, suggesting that the sleep-asthma association was independent of infant wheeze. In fact, the statistical significance for the association increased for nonatopic asthma at age 6 yrs. This was a conservative sensitivity analysis because diminished sleep due to wheeze may well be one of the mechanisms by which early wheeze predisposes children to later asthma.

We also need to acknowledge a number of limitations with the present study. The first relates to the sleep measure. We did not have access to objective information on night awakenings and sleep duration throughout the whole year, or on causes of sleep interruptions, such as snoring or circadian rhythm disorders. Instead, we relied on parent report of night awakenings in their infants for the past week. In addition, lack of temporal data on the occurrence of wheeze versus night awakenings made it difficult for the sensitivity analyses to completely exclude reverse causation. Finally, data were missing for $17 \%$ of children at 6 yrs and for $29 \%$ of children at 14 yrs. Children with missing data were more likely to have asthma risk factors, but these were associated with asthma in the direction expected in study children and did not substantially modify the crude odds ratio. In lieu of the strong association between family stress, nocturnal awakenings and asthma, greater exclusion of children living in high stress families had the potential to cause selection bias. However, the impact of differential loss to follow-up from greater exclusion of children exposed to stress in the night awakenings group would result in a bias towards the null rather than favouring false associations. We deliberately used a very strict definition of asthma, requiring the presence of a physician diagnosis of asthma, current asthma symptoms and current use of asthma medication [24-26]. This definition probably misclassified children with well-controlled asthma and current symptoms as not having asthma, but the misclassification would also have biased results towards the null. It is worth noting that, while nocturnal cough was included as an asthma symptom, no child was classified as having asthma on the basis of nocturnal cough alone.

There is a growing body of literature linking caregiver distress in early life and the development of asthma [50-52]. In the present study, family stress in the post-natal period explained some of the association between frequent nocturnal awakenings during infancy and childhood asthma. Inadequate sleep is more common among children living with parents who argue frequently [2] and family conflict has been reported to precede elevated cortisol levels in children [53]. Sleep deprivation in infants is associated with higher cortisol levels the next morning [10]. Caregiver stress in early life has been associated with increased levels of TNF- $\alpha$ in infants [51]; this proinflammatory cytokine is responsible for sleep control [30]. Recently, in their rat model of a neonatal stressor, FENG et al. [54] documented decreased sleep time and increased levels of hyposecretin in the neonate following separation from the mother. It remains to be confirmed whether sleep deprivation is a cause or outcome of immune system changes, but our evidence suggests that the impact of family stress on the infant's developing immune system could be through its effect on sleep.

In summary, our community-based study of children found an increased risk of nonatopic asthma among children with frequent nocturnal awakenings during the first 3 yrs of life. While our findings are consistent with animal and human studies on the biological effects of sleep deprivation, we consider them hypothesis generating. Further systematic study is required before a direct link between infant sleep and childhood asthma can be made.

\section{SUPPORT STATEMENT}

Funded by National Health and Medical Research Council, Canberra, Australia and the Raine Medical Foundation, University of Western Australia, Perth, Australia.

\section{STATEMENT OF INTEREST}

None declared.

\section{ACKNOWLEDGEMENTS}

Sincere thanks are due to all study families, without whose participation this research could not have been conducted. Many thanks are also extended to the Western Australian Pregnancy Cohort (Raine) study investigators and research staff. The authors wish to acknowledge the Raine Medical Research Foundation, University of Western Australia, Perth, Australia and the National Health and Medical Research Council, Canberra, Australia in funding the establishment and continuation of this birth cohort. Finally, A.L. Kozyrskyj would like to thank M. Berinson and all of the other staff at the Telethon Institute for Child Health Research (TICHR), Perth, for their research support of this project, which was completed by A.L. Kozyrskyj during her research leave at TICHR. The research was conducted independently of the funding agency.

\section{REFERENCES}

1 Webb WB, Agnew HW. Are we chronically sleep deprived? Bull Psychon Soc 1975; 6: 47-48. 
2 Smaldone A, Honig JC, Byrne MW. Sleepless in America: inadequate sleep and relationships to health and well-being of our nation's children. Pediatrics 2007; 119: Suppl. 1, S29-S37.

3 Sadeh A, Pergamin L, Bar-Haim Y. Sleep in children with attention-deficit hyperactivity disorder: a meta-analysis of polysomnographic studies. Sleep Med Rev 2006; 10: 381-398.

4 Gruber R, Grizenko N, Schwartz G, et al. Sleep and COMT polymorphism in ADHD children: preliminary actigraphic data. J Am Acad Child Adolesc Psychiatry 2006; 45: 982-989.

5 Capaldi IV, Handwerger K, Richardson E, et al. Associations between sleep and cortisol responses to stress in children and adolescents: a pilot study. Behav Sleep Med 2005; 3: 177-192.

6 Chaput JP, Brunet M, Tremblay A. Relationship between short sleeping hours and childhood overweight/obesity: results from the "Quebec en Forme" Project. Int J Obes (Lond) 2006; 30: 10801085.

7 Sekine M, Yamagami T, Handa K, et al. A dose-response relationship between short sleeping hours and childhood obesity: results of the Toyama Birth Cohort Study. Child Care Health Dev 2002; 28: 163-170.

8 Salzarulo P, Fagioli I. Sleep for development or development for waking? Some speculations from a human perspective. Behav Brain Res 1995; 69: 23-27.

9 Watamura SE, Donzella B, Kertes DA, et al. Developmental changes in baseline cortisol activity in early childhood: relations with napping and effortful control. Dev Psychobiol 2004; 45: 125133.

10 Silva ML, Mallozi MC, Ferrari GF. Salivary cortisol to assess the hypothalamic-pituitary-adrenal axis in healthy children under 3 years old. J Pediatr (Rio J) 2007; 83: 121-126.

11 Obal F Jr, Krueger JM. GHRH and sleep. Sleep Med Rev 2004; 8: 367-377.

12 Anders TF. Biological rhythms in development. Psychosom Med 1982; 44: 61-72.

13 Baum WF, Schneyer U, Lantzsch AM, et al. Delay of growth and development in children with bronchial asthma, atopic dermatitis and allergic rhinitis. Exp Clin Endocrinol Diabetes 2002; 110: 53-59.

14 Ball TM, Anderson D, Minto J, et al. Cortisol circadian rhythms and stress responses in infants at risk of allergic disease. J Allergy Clin Immunol 2006; 117: 306-311.

15 Buske-Kirschbaum A, Fischbach S, Rauh W, et al. Increased responsiveness of the hypothalamus-pituitary-adrenal (HPA) axis to stress in newborns with atopic disposition. Psychoneuroendocrinology 2004; 29: 705-711.

16 Buske-Kirschbaum A, von Auer K, Krieger S, et al. Blunted cortisol responses to psychosocial stress in asthmatic children: a general feature of atopic disease? Psychosom Med 2003; 65: 806-810.

17 Ising $\mathrm{H}$, Lange-Asschenfeldt $\mathrm{H}$, Moriske $\mathrm{HJ}$, et al. Low frequency noise and stress: bronchitis and cortisol in children exposed chronically to traffic noise and exhaust fumes. Noise Health 2004; 6: 21-28.

18 Stein MA, Mendelsohn J, Obermeyer WH, et al. Sleep and behavior problems in school-aged children. Pediatrics 2001; 107: E60.

19 Newnham JP, Evans SF, Michael CA, et al. Effects of frequent ultrasound during pregnancy: a randomised controlled trial. Lancet 1993; 342: 887-891.

20 Touchette E, Petit D, Paquet J, et al. Factors associated with fragmented sleep at night across early childhood. Arch Pediatr Adolesc Med 2005; 159: 242-249.

21 Scher A. Infant sleep at 10 months of age as a window to cognitive development. Early Hum Dev 2005; 81: 289-292.

22 Sekine M, Chen X, Hamanishi S, et al. The validity of sleeping hours of healthy young children as reported by their parents. J Epidemiol 2002; 12: 237-242.

23 Sadeh A. A brief screening questionnaire for infant sleep problems: validation and findings for an internet sample. Pediatrics 2004; 113: e570-e577.
24 Joseph-Bowen J, de Klerk NH, Firth MJ, et al. Lung function, bronchial responsiveness, and asthma in a community cohort of 6year-old children. Am J Respir Crit Care Med 2004; 169: 850-854.

25 Joseph-Bowen J, de Klerk N, Holt PG, et al. Relationship of asthma, atopy, and bronchial responsiveness to serum eosinophil cationic proteins in early childhood. J Allergy Clin Immunol 2004; 114: 10401045.

26 Morahan G, Huang D, Wu M, et al. Association of IL12B promoter polymorphism with severity of atopic and non-atopic asthma in children. Lancet 2002; 360: 455-459.

27 Tennant $C$, Andrews G. A scale to measure the stress of life events Aust N Z J Psychiatry 1976; 10: 27-32.

28 Wright AL. Epidemiology of asthma and recurrent wheeze in childhood. Clin Rev Allergy Immunol 2002; 22: 33-44.

29 Markov D, Goldman M. Normal sleep and circadian rhythms: neurobiologic mechanisms underlying sleep and wakefulness. Psychiatr Clin North Am 2006; 29: 841-853.

30 Siegel JM. Clues to the functions of mammalian sleep. Nature 2005; 437: 1264-1271.

31 Steinman L. Elaborate interactions between the immune and nervous systems. Nat Immunol 2004; 5: 575-581.

32 Irwin MR, Wang M, Campomayor CO, et al. Sleep deprivation and activation of morning levels of cellular and genomic markers of inflammation. Arch Intern Med 2006; 166: 1756-1762.

33 Okun ML, Coussons-Read ME. Sleep disruption during pregnancy: how does it influence serum cytokines? J Reprod Immunol 2007; 73: 158-165.

34 Okun ML, Hall M, Coussons-Read ME. Sleep disturbances increase interleukin-6 production during pregnancy: implications for pregnancy complications. Reprod Sci 2007; 14: 560-567.

35 Silvestri M, Bontempelli M, Giacomelli M, et al. High serum levels of tumour necrosis factor- $\alpha$ and interleukin- 8 in severe asthma: markers of systemic inflammation? Clin Exp Allergy 2006; 36: 1373-1381.

36 Li AM, Hung E, Tsang T, et al. Induced sputum inflammatory measures correlate with disease severity in children with obstructive sleep apnoea. Thorax 2007; 62: 75-79.

37 Balfour-Lynn IM, Valman HB, Wellings R, et al. Tumour necrosis factor- $\alpha$ and leukotriene E4 production in wheezy infants. Clin Exp Allergy 1994; 24: 121-126.

38 Heaton T, Rowe J, Turner S, et al. An immunoepidemiological approach to asthma: identification of in vitro T-cell response patterns associated with different wheezing phenotypes in children. Lancet 2005; 365: 142-149.

39 Steiger A. Neurochemical regulation of sleep. J Psychiatr Res 2007; 41: 537-552.

40 Lambrecht BN. Immunologists getting nervous: neuropeptides, dendritic cells and T cell activation. Respir Res 2001; 2: 133-138.

41 Horne RS, Ferens D, Watts AM, et al. Effects of maternal tobacco smoking, sleeping position, and sleep state on arousal in healthy term infants. Arch Dis Child Fetal Neonatal Ed 2002; 87: F100-F105.

42 Wolke D, Meyer R, Ohrt B, et al. The incidence of sleeping problems in preterm and fullterm infants discharged from neonatal special care units: an epidemiological longitudinal study. J Child Psychol Psychiatry 1995; 36: 203-223.

43 Pekkanen J, Pearce N. Defining asthma in epidemiological studies. Eur Respir J 1999; 14: 951-957.

44 Martinez FD. What have we learned from the Tucson Children's Respiratory Study? Paediatr Respir Rev 2002; 3: 193-197.

45 Ozdemir C, Ceyhan BB, Yazi D, et al. Non-atopic asthma in children is related to maternal bronchial hyperreactivity. Pediatr Allergy Immunol 2008; 19: 248-254.

46 Kurukulaaratchy RJ, Fenn M, Matthews S, et al. Characterisation of atopic and non-atopic wheeze in 10 year old children. Thorax 2004; 59: 563-568.

47 Reuveni H, Chapnick G, Tal A, et al. Sleep fragmentation in children with atopic dermatitis. Arch Pediatr Adolesc Med 1999; 153: 249-253. 
48 Turner-Warwick M. Epidemiology of nocturnal asthma. Am J Med 1988; 85: 6-8.

49 Walton RP, Johnston SL. Role of respiratory viral infections in the development of atopic conditions. Curr Opin Allergy Clin Immunol 2008; 8: 150-153.

50 Kozyrskyj AL, Mai XM, McGrath P, et al. Continued exposure to maternal distress in early life is associated with an increased risk of childhood asthma. Am J Respir Crit Care Med 2008; 177: 142-147.

51 Wright RJ, Finn P, Contreras JP, et al. Chronic caregiver stress and IgE expression, allergen-induced proliferation, and cytokine profiles in a birth cohort predisposed to atopy. J Allergy Clin Immunol 2004; 113: 1051-1057.

52 Klinnert MD, Nelson HS, Price MR, et al. Onset and persistence of childhood asthma: predictors from infancy. Pediatrics 2001; 108: E69.

53 Waynforth D. The influence of parent-infant cosleeping, nursing, and childcare on cortisol and SIgA immunity in a sample of British children. Dev Psychobiol 2007; 49: 640-648.

54 Feng P, Vurbic D, Wu Z, et al. Brain orexins and wake regulation in rats exposed to maternal deprivation. Brain Res 2007; 1154: 163-172. 